Determination of Organic Partitioning Coefficients in Water-Supercritical $\mathrm{CO}_{2}$ Systems by Simultaneous In Situ UV and Near-Infrared Spectroscopies

David A. Bryce, ${ }^{\dagger}$ Hongbo Shao, ${ }^{\dagger}$ Kirk J. Cantrell, and Christopher J. Thompson ${ }^{*}$

Pacific Northwest National Laboratory, P.O. Box 999, Richland, WA 99352, USA

6 Summary

7 Three SI pages containing one figure, a brief discussion of $\mathrm{CO}_{2}$ density trends, and a reference. 


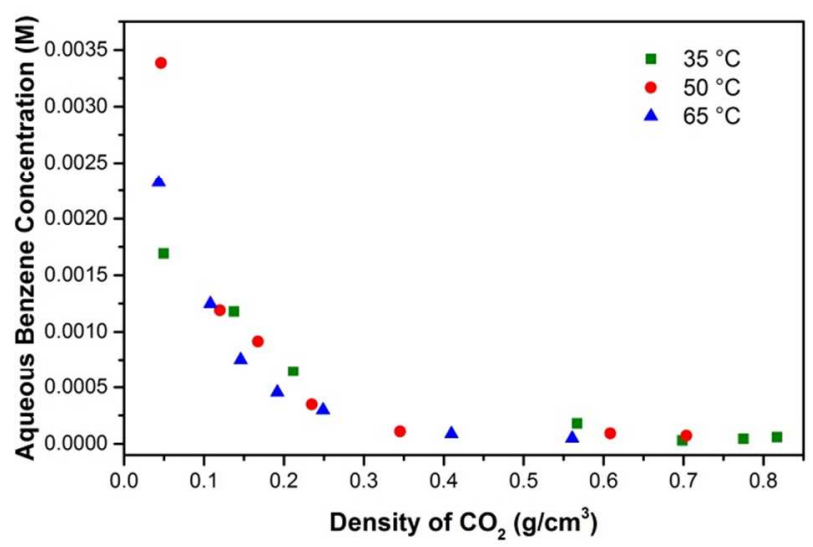

10 Figure S1. Equilibrium aqueous benzene concentrations measured in this study as a function of

$11 \mathrm{CO}_{2}$ phase density.

\section{$12 \mathrm{CO}_{2}$ Density Variation with Pressure.}

13 Figure 5 (main manuscript) shows density trends for $\mathrm{CO}_{2}$ as a function of pressure for the

14 temperatures used in this study. At low pressures corresponding to the gas phase realm, there are

15 limited changes in density as the pressure is increased. However, as the critical point is

16 approached, the density increases rapidly with rising pressure until the conditions are well into

17 the supercritical range, at which point smaller changes in density are observed as the pressure is

18 further increased. Additionally, qualitative comparison of the trends in density between the

19 plotted temperatures indicates that the dramatic density increase near the critical point is less

20 pronounced in the higher temperature data $\left(65^{\circ} \mathrm{C}\right)$ than in the lower temperature profiles. At high

21 temperatures, the density-pressure relationship approaches linearity while at lower temperatures,

22 the profile resembles a sigmoid function. The dependence of solubility on supercritical fluid

23 density has been established previously, ${ }^{1}$ and it follows that density should be one of the major

24 factors which influence partitioning of compounds of interest between water and $\mathrm{scCO}_{2}$. 
27 1. Chrastil, J., Solubility of solids and liquids in supercritical gases. The Journal of Physical 28 Chemistry 1982, 86, (15), 3016-3021. 